\title{
Distribuição de Metais Pesados nos Sedimentos de Fundo ao Longo da Bacia do Rio Tietê
}

\author{
Heavy Metal Distribution in the Bottom Sediments Along Tietê River Basin
}

\author{
Jefferson Mortatti' (jmortatt@cena.usp.br), Christophe Hissler² (hissler@lippmann.lu), \\ Jean-Luc Probst ${ }^{3}$ (jean-luc.probst@ensat.fr) \\ 'Centro de Energia Nuclear na Agricultura - ESALQ - USP \\ Av. Centenário 303, CEP 13416-970, Piracicaba, SP, BR \\ ${ }^{2}$ Environment and Biotechnologies - CRC Gabriel Lippmann, Luxembourg, LU \\ ${ }^{3}$ Ecolab - École Nationale Supérieure Agronomique de Toulouse - INP, Toulouse, FR
}

Recebido em 10 de junho de 2009; aceito em 08 de janeiro de 2010

\section{RESUMO}

Análises de metais pesados em sedimentos fluviais têm sido utilizadas para um melhor entendimento das cargas críticas e grau de toxicidade desses metais em função das influências antrópicas em bacias de drenagem, principalmente relacionados aos efluentes domésticos, industriais e agrícolas. O presente estudo reporta a distribuição de $\mathrm{Cr}, \mathrm{Cu}, \mathrm{Ni}, \mathrm{Zn}$ e $\mathrm{Pb}$ nos sedimentos de fundo ao longo da bacia do rio Tietê, uma das regiões mais poluídas do sudeste do Brasil. Na parte alta da bacia de drenagem, as concentrações dos metais pesados se mostraram distribuídas na seguinte ordem decrescente $\mathrm{Zn}>$ $\mathrm{Cr}>\mathrm{Pb}>\mathrm{Ni}>\mathrm{Cu}$, enquanto que a jusante, na parte média da bacia (região sob forte influência antrópica) elevadas concentrações de Zn, Cu e Cr com 648, 200 e 183 g g-1, respectivamente, puderam ser observadas. O potencial de biodisponibilidade dos metais pesados extraídos dos sedimentos de fundo do rio Tietê foi avaliado em função da análise por extração sequencial utilizada, a qual mostrou que na parte alta da bacia, a maioria dos metais pesados se mostrou mais concentrado na fração residual dos sedimentos, enquanto que à jusante, $\mathrm{Zn}, \mathrm{Cr}$ e Ni se mostraram associados à fração biodisponível em mais de $60 \%$, sendo ainda principalmente ligados aos óxidos de Fe. As elevadas concentrações observadas na parte média da bacia de drenagem, quando comparadas com as concentrações médias do fundo geoquímico natural, se mostraram em termos do fator de enriquecimento e índice de geoacumulação, fortemente poluídas para Zn e de moderada para fortemente poluída para $\mathrm{Ni}$, no sentido da foz.

Palavras-chave: Rio Tietê; Metal pesado; Sedimento de fundo; Extração sequencial; EF; $I_{\text {geo }}$.

\begin{abstract}
Analysis of heavy metals in fluvial sediments has been used to better understand the potential hazards and critical toxicity of heavy metals mainly related to the anthropogenic influences of urban sewage, industrial effluents and agricultural activity. The present study analyzed the heavy metals, such as $\mathrm{Cr}, \mathrm{Cu}, \mathrm{Ni}, \mathrm{Zn}$ and $\mathrm{Pb}$, in the bottom sediments along the Tietê River basin, a polluted watershed in the Southeast region of Brazil. The distribution of the concentrations of heavy metals in the upper basin decreased in the following order: $\mathrm{Zn}>\mathrm{Cr}>\mathrm{Pb}>\mathrm{Ni}>\mathrm{Cu}$, whereas, in the middle part of the basin (which is under strong urban and industrial influences), high concentrations of $\mathrm{Zn}, \mathrm{Cu}$ and $\mathrm{Cr}$ were observed, measuring 648, 200 and $183 \mu \mathrm{g} g-1$, respectively. The sequential chemical extraction in relation to the total concentration was used to assess the potential bioavailability of heavy metals. In the upper part of the basin, the heavy metals extracted from the bottom sediments were mainly related to the residual fraction, whereas, in the middle part of the basin, the bioavailability of $\mathrm{Zn}, \mathrm{Cr}$ and $\mathrm{Ni}$ was higher than $60 \%$, and these metals were mainly related to the iron oxide phase. The high concentration of heavy metals observed in the middle basin of the Tietê river (after the metropolitan area of São Paulo), when compared with the average concentration found in the geochemical bottom, showed that, in terms of enrichment factor and geoaccumulation index, the degree of pollution by $\mathrm{Zn}$ is high and by $\mathrm{Ni}$ is high to moderate in the downstream direction.
\end{abstract}

Keywords: Tietê River; Heavy metal; Bottom sediment; Sequential extraction; EF; $I_{\text {geo }}$. 


\section{INTRODUÇÃO}

O Tietê é o mais importante rio do Estado de São Paulo devido principalmente ao seu potencial hidroelétrico, o qual é bem explorado em quase toda a sua extensão de $1.100 \mathrm{~km}$. Com o crescimento populacional e industrial da cidade de São Paulo, o rio Tietê passou a ser fortemente influenciado pela geração, sem controle, de esgotos domésticos e industriais, deteriorando a qualidade de suas águas e sedimentos.

Os metais pesados estão presentes entre os principais poluentes originários dessas atividades antrópicas, podendo ser encontrados sob diferentes formas químicas nos principais compartimentos do ambiente (atmosfera, água, solos, sedimentos e organismos vivos), sempre associados a um alto fator de enriquecimento e a baixas taxas de remoção (Elderfield, Upstill-Goddard, Sholkovitz, 1990; Sholkovitz, 1992; Mortatti et al., 2002). Estudos geoquímicos dos sedimentos fluviais, normalmente conduzidos em amostras de sedimento de fundo, permitem uma avaliação histórica da origem e distribuição dos metais pesados na área de drenagem.

As distribuições e quantificações dos metais pesados em sedimentos fluviais são normalmente baseadas nas concentrações obtidas a partir das extrações totais e comparadas com a caracterização do fundo geoquímico natural (Salomons e Forstner, 1984), enquanto que procedimentos químicos mais detalhados, como as extrações sequenciais, permitem a identificação das frações associadas às complexações metálicas possíveis.

$\mathrm{O}$ presente trabalho teve por objetivo avaliar a distribuição dos metais pesados $\mathrm{Cr}, \mathrm{Cu}, \mathrm{Ni}, \mathrm{Zn}$ e $\mathrm{Pb}$ nos sedimentos de fundo ao longo da bacia do rio Tietê, desde a nascente até a sua parte média superior junto à barragem de Barra Bonita, identificando os possíveis impactos antrópicos em termos dos fatores de enriquecimento de metais pesados e classes de poluição.

\section{ÁREA DE ESTUDO}

A área de estudo compreende a bacia de drenagem do rio Tietê com $32.930 \mathrm{~km}^{2}$, desde a nascente até o reservatório de Barra Bonita, estando aproximadamente a $260 \mathrm{~km}$ do Oceano Atlântico (Figura 1). A nascente do rio Tietê localiza-se na região de Salesópolis, numa altitude de $840 \mathrm{~m}$, e sua calha principal cruza a área metropolitana da cidade de São Paulo, com uma pressão urbana de cerca de 20 milhões de habitantes, recebendo uma enorme quantidade de esgotos domésticos e industriais sem tratamento prévio.

Em sua maior parte, a bacia do rio Tietê apresenta rochas cristalinas e metamórficas associadas à zona de Depressão Periférica, sendo que somente mais à jusante é que se apresentam rochas sedimentares detríticas e pequenas regiões de basalto. De acordo com Oliveira (1999) os principais tipos de solos encontrados ao longo da bacia de drenagem são os ultissolos $(55,4 \%)$ e oxissolos $(28,3 \%)$, os quais cobrem uma área de intensa atividade agrícola (principalmente cana-de-açúcar) e industrial (papel, celulose, produtos têxteis e refinarias de petróleo).

A bacia do rio Tietê é caracterizada pelo tipo climático Cwa, de acordo com a classificação de Köppen, com inverno seco e verão chuvoso quente.

\section{MATERIAL E MÉTODOS}

Os sedimentos de fundo ao longo do rio Tietê foram amostrados no período de março a junho de 2004, com a utilização de uma draga manual, correspondendo a uma profundidade média de $5 \mathrm{~cm}$, em três locais distintos no mesmo ponto de amostragem, visando uma melhor representatividade amostral, sendo as amostras compostas posteriormente. Os pontos de amostragem dos sedimentos de fundo ao longo do rio Tietê, conforme pode ser observado na Figura1, foram os seguintes: $\mathrm{T} 0$ - junto à nascente em Salesópolis; T1 - Mogi das Cruzes; T2 - Santana do Parnaíba; T3 - Tietê; T4 - Anhembi; T5 - junto à entrada da represa de Barra Bonita; T6 - no meio da represa de Barra Bonita e T7 - junto à barragem de Barra Bonita. As amostras foram acondicionadas em recipientes plásticos, secas ao ar, homogeneizadas e em seguida processadas em peneiras de fibra de nylon, a fim de separar a fração inferior a $63 \mu \mathrm{m}$ (siltes e argilas), de acordo com Förstner e Salomons (1980).

As análises químicas dos metais pesados $\mathrm{Cr}, \mathrm{Cu}, \mathrm{Ni}$, $\mathrm{Zn}$ e $\mathrm{Pb}$ foram realizadas usando um ICP OES, após extração total de $100 \mathrm{mg}$ de amostra por fusão alcalina, com tetraborato e metaborato de lítio (2:1), em cadinhos de platina a $1.000^{\circ} \mathrm{C}$ por $30 \mathrm{~min}$, em triplicata (Samuel, Roult, Besnus, 1985).

Para controle da qualidade analítica e do processo de extração, foi utilizado o material de referência internacional Soil-7 (IAEA), sendo obtidos os seguintes porcentuais médios de recuperação para extração e análise em triplicata: $\mathrm{Cr}(92,3 \pm 5,1 \%), \mathrm{Cu}(94,1 \pm 3,2 \%), \mathrm{Co}(95,3 \pm 6,4 \%)$, $\mathrm{Ni}(97,4 \pm 3,4 \%), \mathrm{Zn}(103 \pm 9,2 \%)$ e $\mathrm{Pb}(107,1 \pm 4,8 \%)$.

A extração sequencial seletiva dos metais pesados $\mathrm{Cr}$, $\mathrm{Cu}, \mathrm{Ni}, \mathrm{Zn}$ e $\mathrm{Pb}$ dos sedimentos de fundo ao longo do rio Tietê foi realizada de acordo com o procedimento descrito por Leleyter e Probst (1999), o qual permite identificar cada uma das frações da fase lábil, mudando as composições físico-químicas das fases aquosas com o emprego de soluções químicas distintas (Tabela 1).

A soma de todas as frações lixiviáveis (S1 a S6) representa nesse método a fração lábil ou biodisponível do sedimento fluvial. 


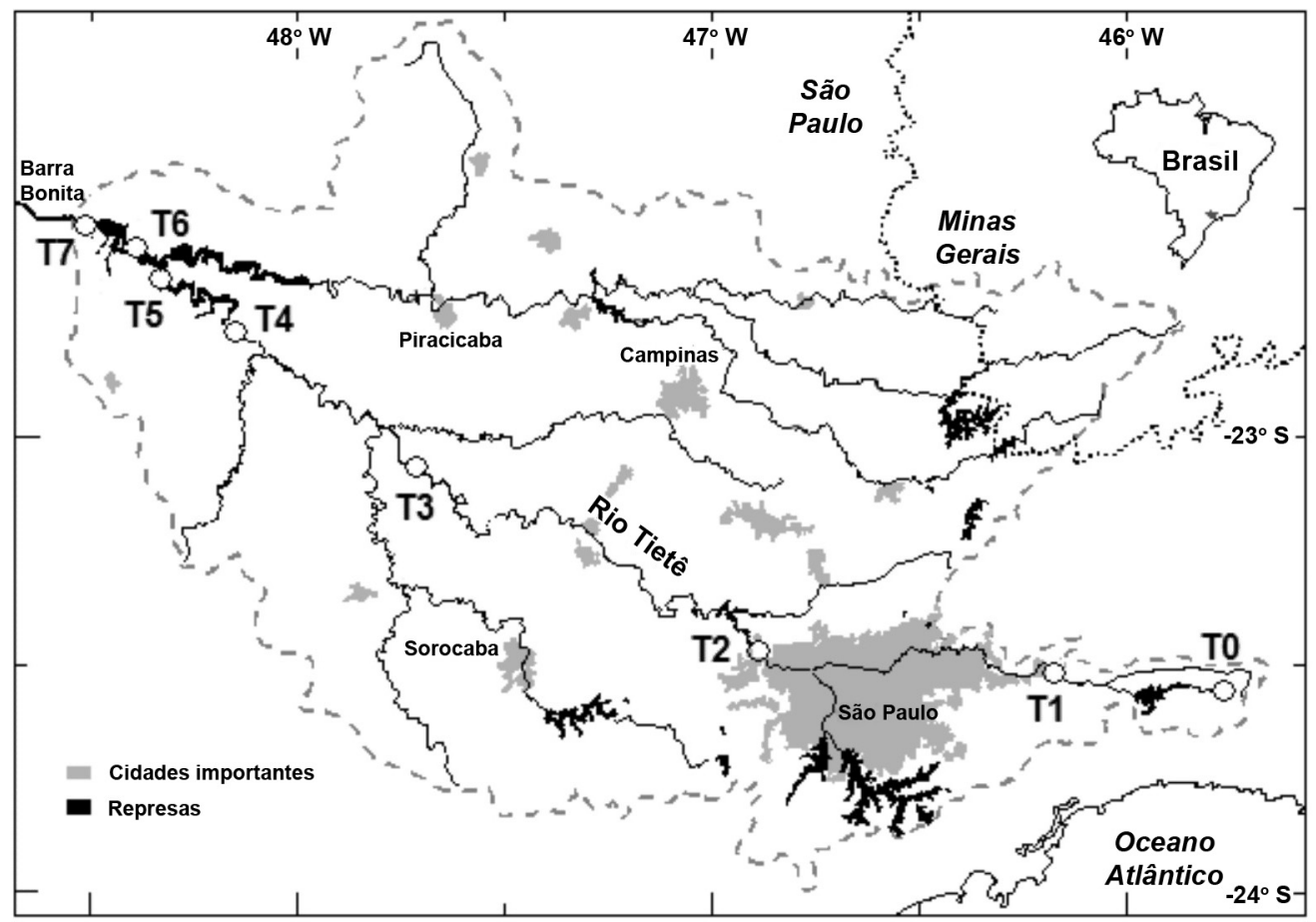

Figura 1. Localização da bacia do rio Tietê e pontos de amostragem dos sedimentos de fundo.

Tabela 1. Condições das reações químicas para a extração sequencial seletiva de metais pesados nos sedimentos de fundo do rio Tietê (Leleyter e Probst, 1999).

\begin{tabular}{|c|c|c|c|c|c|c|}
\hline & Fração & Extrator & & Tempo (h) & $\mathrm{T}\left({ }^{\circ} \mathrm{C}\right)$ & $\mathrm{pH}$ \\
\hline S1 & solúvel & $10 \mathrm{~mL}$ água Milli-Q & & 0,5 & 20 & 5,7 \\
\hline S2 & trocável & $10 \mathrm{~mL} \mathrm{Mg}\left(\mathrm{NO}_{3}\right)_{2} 1 \mathrm{M}$ & & 2 & 20 & 5,0 \\
\hline S3 & ácido solúvel & $10 \mathrm{~mL} \mathrm{NaOAc} 1 \mathrm{M}$ & & 5 & 20 & 4,5 \\
\hline S4 & óxido $\mathrm{Mn}$ & $10 \mathrm{~mL} \mathrm{NH}_{2} \mathrm{OH} \mathrm{HCl} \quad 0,1 \mathrm{M}$ & & 0,5 & 20 & 3,5 \\
\hline \multirow{2}{*}{ S5 } & \multirow{2}{*}{ óxido Fe } & $10 \mathrm{~mL}\left(\mathrm{NH}_{4}\right)_{2} \mathrm{C}_{2} \mathrm{O}_{4} \quad 0,2 \mathrm{M}+\mathrm{H}_{2} \mathrm{C}_{2} \mathrm{O}_{4} \quad 0,2 \mathrm{M}$ & & 4 & 20 & 3,0 \\
\hline & & $10 \mathrm{~mL}\left(\mathrm{NH}_{4}\right)_{2} \mathrm{C}_{2} \mathrm{O}_{4} \quad 0,2 \mathrm{M}+\mathrm{H}_{2} \mathrm{C}_{2} \mathrm{O}_{4} \quad 0,2 \mathrm{M}+\mathrm{C}_{6} \mathrm{H}_{8} \mathrm{O}_{6}$ & $0,1 \mathrm{M}$ & 0,5 & 80 & 2,3 \\
\hline \multirow[t]{2}{*}{ S6 } & mat. orgânica & $3 \mathrm{~mL} \mathrm{HNO}_{3} \quad 0,02 \mathrm{M}+8 \mathrm{~mL} \quad 35 \% \quad \mathrm{H}_{2} \mathrm{O}_{2}$ & & 5 & 85 & 2,0 \\
\hline & & $5 \mathrm{~mL} \mathrm{NH} \mathrm{H}_{4} \mathrm{OAc} \quad 3,2 \mathrm{M}+10 \mathrm{~mL}$ água Milli-Q & & 0,5 & 20 & \\
\hline
\end{tabular}

O procedimento de extração dos metais pesados dos sedimentos de fundo ao longo do rio Tietê foi o seguinte: colocação de $1 \mathrm{~g}$ de sedimento seco ao ar, fração fina $(<63 \mu \mathrm{m})$ em um recipiente de teflon juntamente com a respectiva solução extratora, sob agitação contínua, seguindo o protocolo estabelecido para cada extração de acordo com a Tabela 1. No fim de cada etapa de extração, o resíduo foi filtrado a $0,45 \mu \mathrm{m}$ (filtragem manual a vácuo com filtro Millipore HVLP) e em seguida lavado com água destilada Milli-Q, seco em estufa a $40^{\circ} \mathrm{C}$ e preparado para a nova etapa da ex- tração. As análises químicas dos metais pesados $\mathrm{Cr}, \mathrm{Cu}, \mathrm{Ni}$, $\mathrm{Zn}$ e $\mathrm{Pb}$, em cada fase das extrações, foram realizadas usando um ICP-OES.

As intensidades das possíveis anomalias de $\mathrm{Cr}, \mathrm{Cu}, \mathrm{Ni}$, $\mathrm{Zn}$ e $\mathrm{Pb}$ nos sedimentos de fundo ao longo do rio Tietê foram expressas utilizando o fator de enriquecimento (EF) definido por Gresens (1967), tendo o Sc como elemento normalizador, de acordo com a equação 1 :

$$
\mathrm{EF}=\left(\mathrm{M}_{\mathrm{i}} / \mathrm{Sc}\right)_{\mathrm{am}} /\left(\mathrm{M}_{\mathrm{i}} / \mathrm{Sc}\right)_{\mathrm{ref}}
$$


onde $\left(\mathrm{M}_{\mathrm{i}} / \mathrm{Sc}\right)_{\mathrm{am}}$ representa a razão entre a concentração do metal pesado de interesse e o Sc na amostra e $\left(\mathrm{M}_{\mathrm{i}} / \mathrm{Sc}\right)_{\text {ref }}$ a mesma razão em relação ao fundo geoquímico natural de referência.

O índice de geoacumulação $\left(I_{\text {geo }}\right)$, introduzido por Müller (1979), foi utilizado para a avaliação das classes de poluição dos metais pesados nos sedimentos de fundo ao longo do rio Tietê durante o período estudado (equação 2):

$$
I_{\text {geo }}=\log _{2}[\mathrm{Me}]_{\text {sed }} / 1.5[\mathrm{Me}]_{\text {ref }}
$$

onde, $[\mathrm{Me}]_{\text {sed }}$ representa a concentração do metal pesado no sedimento e $[\mathrm{Me}]_{\text {ref }}$ a concentração do metal pesado relativo ao fundo geoquímico natural de referência.

O índice $I_{\text {geo }}$ utilizado apresenta sete classes de poluição em função dos graus de 0 (ausência de poluição) a 6 (poluição muito forte).

A caracterização do fundo geoquímico natural na bacia de drenagem do rio Tietê foi realizada a partir das concentrações dos metais pesados presentes nos sedimentos de fundo na parte alta da bacia de drenagem, sem qualquer influência antrópica, obtidos por Favaro et al. (2004), com concentrações médias para $\mathrm{Cr}, \mathrm{Cu}, \mathrm{Ni}, \mathrm{Zn}, \mathrm{Pb}$ e $\mathrm{Sc}$ de 40, $50,28,50,30$ e $14 \mu \mathrm{g} \mathrm{g}^{-1}$, respectivamente.

Em termos comparativos, também foram utilizados os dados médios das concentrações desses metais pesados presentes na crosta terrestre, de acordo com Taylor e McLennan (1985), com concentrações médias para $\mathrm{Cr}, \mathrm{Cu}, \mathrm{Ni}, \mathrm{Zn}, \mathrm{Pb}$ e Sc de 35, 25, 20, 71, 20 e $11 \mu \mathrm{g} \mathrm{g}^{-1}$, respectivamente.

\section{RESULTADOS E DISCUSSÃO}

\section{Concentração total dos metais pesados}

A Tabela 2 apresenta os principais resultados da extração total dos metais pesados estudados na bacia do rio Tietê, desde a nascente em Salesópolis (T0) até o reservatório de Barra Bonita (T7), incluindo ainda as concentrações médias dos metais pesados junto ao fundo geoquímico natural e as concentrações médias desses metais na crosta terrestre.

Foi possível observar, para a maioria dos metais pesados identificados nos sedimentos de fundo do rio Tietê, elevadas concentrações junto às estações de Santana do Parnaíba (T2) e Tietê (T3), regiões à jusante da zona metropolitana, conhecida como "grande São Paulo", receptoras de grande carga de efluentes urbanos sem tratamento prévio. As concentrações verificadas em $\mathrm{T} 2$ para $\mathrm{Zn}, \mathrm{Cu}$ e $\mathrm{Cr}$ foram de 648,200 e $183 \mu \mathrm{g} \mathrm{g}^{-1}$, respectivamente, enquanto que para $\mathrm{Ni}$ e $\mathrm{Pb}$, as concentrações foram de 92 e $77 \mu \mathrm{g} \mathrm{g}^{-1}$, respectivamente. Tais valores excederam em muito as concentrações observadas para o fundo geoquímico natural e a composição média da crosta terrestre. Em termos comparativos, tais concentrações, especialmente para $\mathrm{Zn} \mathrm{e} \mathrm{Cu}$, foram cerca de 10 vezes superiores às verificadas na bacia do rio Huaihe na China, a qual apresenta problemas antrópicos similares (Yan, Yong, Huang, 2007).

Ainda à jusante, as concentrações da maioria dos metais pesados nos sedimentos de fundo amostrados diminuí-

Tabela 2. Concentrações médias dos metais pesados nos sedimentos de fundo ao longo da bacia do rio Tietê, desde a nascente em Salesópolis (T0) até o reservatório de Barra Boniła (T7), com os respectivos desvios, incluindo as composições médias do fundo geoquímico natural e da crosta terrestre. a. Composição média da crosta terrestre (Taylor e McLennan, 1985). b. Sedimento de fundo natural (Favaro et al., 2004).

\begin{tabular}{|c|c|c|c|c|c|c|}
\hline \multirow{2}{*}{ Local } & $\mathrm{Cr}$ & $\mathrm{Cu}$ & $\mathrm{Ni}$ & $\mathrm{Zn}$ & $\mathbf{P b}$ & Sc \\
\hline & \multicolumn{6}{|c|}{$\mu \mathrm{g} \mathrm{g}^{-1}$} \\
\hline TO & $60 \pm 3$ & $41 \pm 2$ & $43 \pm 3$ & $88 \pm 4$ & $46 \pm 3$ & $16 \pm 1$ \\
\hline $\mathrm{T} 1$ & $114 \pm 3$ & $53 \pm 2$ & $31 \pm 2$ & $266 \pm 9$ & $47 \pm 2$ & $19 \pm 1$ \\
\hline $\mathrm{T} 2$ & $183 \pm 8$ & $200 \pm 8$ & $92 \pm 8$ & $648 \pm 28$ & $77 \pm 4$ & $19 \pm 1$ \\
\hline T3 & $133 \pm 4$ & $79 \pm 4$ & $60 \pm 3$ & $560 \pm 32$ & $44 \pm 3$ & $16 \pm 1$ \\
\hline $\mathrm{T} 4$ & $127 \pm 2$ & $97 \pm 4$ & $67 \pm 1$ & $381 \pm 14$ & $36 \pm 4$ & $14 \pm 1$ \\
\hline T5 & $132 \pm 8$ & $46 \pm 3$ & $80 \pm 4$ & $266 \pm 18$ & $30 \pm 2$ & $15 \pm 2$ \\
\hline T6 & $84 \pm 5$ & $39 \pm 2$ & $70 \pm 5$ & $163 \pm 11$ & $18 \pm 2$ & $12 \pm 1$ \\
\hline$T 7$ & $262 \pm 3$ & $55 \pm 1$ & $51 \pm 2$ & $514 \pm 21$ & $18 \pm 1$ & $14 \pm 1$ \\
\hline${ }^{\mathrm{a}}$ crosta & 35 & 25 & 20 & 71 & 20 & 11 \\
\hline${ }^{\mathrm{b}} \mathrm{FN}_{\text {sed }}$ & 40 & 50 & 28 & 50 & 30 & 14 \\
\hline
\end{tabular}


ram significativamente até a entrada da represa em Barra Bonita (T6), aumentando posteriormente para $\mathrm{Zn}, \mathrm{Cr}$ e $\mathrm{Cu}$ junto à barragem final (T7). Tal ocorrência pode estar associada às zonas de maior turbulência do canal fechado próximas à hidroelétrica.

\section{Extração sequencial seletiva}

A Figura 2 apresenta os principais resultados da extração sequencial seletiva dos metais pesados presentes nos sedimentos de fundo ao longo do rio Tietê, utilizada no presente trabalho. Pode ser verificado que, próximo às nascentes, na parte alta da bacia de drenagem (T0 e T1), a maioria dos metais estudados se encontra na fração residual dos sedimentos, com exceção do $\mathrm{Cu}$, que se mostrou na fração biodisponível com cerca de $70 \%$, sendo ligada às fases orgânica e óxido de $\mathrm{Fe}$, preferencialmente.

Para as estações de amostragens em Santana do Parnaíba (T2) e Tietê (T3), o caráter de biodisponibilidade aumenta acentuadamente $(>60 \%)$ para todos os metais, indicando ligações principalmente associadas à fase óxido de $\mathrm{Fe}$, com exceção do $\mathrm{Cu}$, que se mantém ligado à matéria orgânica. Em proporção menor, $\mathrm{Zn}$ e $\mathrm{Pb}$ se mostraram também associados à fase de carbonatos ou ácido solúvel. Resultados similares foram apresentados por Gomez et al. (2007) para o rio San Pedro, na cidade de Sonora, México, onde a influência da área urbana na qualidade dos sedimentos de fundo foi também detectada, sendo as fases de óxidos de $\mathrm{Fe}$ e $\mathrm{Mn}$ as principais em ligação com $\mathrm{Pb}$ e $\mathrm{Zn}$.

Com relação às estações de amostragens mais à jusante na bacia de drenagem, principalmente junto à represa de Barra Bonita (pontos T6 e T7), o caráter de biodisponibilidade diminui acentuadamente para a maioria dos metais pesados estudados, indicando que esses metais se encontram na fase residual dos sedimentos. $\mathrm{O} \mathrm{Pb}$, de maneira contrária, se mostra preferencialmente associado à fase óxido de $\mathrm{Fe}$ e consequentemente biodisponível (> 80\%). Tal resultado se mostrou de acordo com o verificado por Mortatti et al. (2002) para a bacia do rio Piracicaba, pertencente a mesma bacia do rio Tietê, onde o $\mathrm{Pb}$ se mostrou cerca de $90 \%$ ligado ao óxido de $\mathrm{Fe}$, para sedimentos em suspensão amostrados junto à estação de Artemis.

O padrão de distribuição dos metais pesados $\mathrm{Cr}, \mathrm{Cu}, \mathrm{Ni}$, $\mathrm{Zn}, \mathrm{Pb}$ nas frações residual e biodisponível mostra uma predominância dos mesmos para a fração biodisponível, sensível aos aportes antrópicos, principalmente devido às influências da grande metrópole de São Paulo, que atingem diretamente as estações de Santana do Parnaíba (T2), essencialmente efluentes domésticos e industriais, e Tietê (T3), com aportes agrícolas também significantes. Resultados similares foram obtidos por Molisani, Salomão e Olivalle (2005) devido aos aportes antrópicos na bacia do rio Paraí- ba do Sul, principalmente em direção à zona estuarina, onde a fração biodisponível se mostrou superior a $80 \%$.

\section{Fator de enriquecimento}

A Tabela 3 apresenta os resultados das possíveis anomalias de $\mathrm{Cr}, \mathrm{Cu}, \mathrm{Ni}, \mathrm{Zn}$ e $\mathrm{Pb}$ nos sedimentos de fundo ao longo do rio Tietê, expressas de acordo com o fator de enriquecimento (EF) calculado tendo o Sc como elemento normalizador, considerando o fundo geoquímico natural da bacia de drenagem e a composição média da crosta terrestre.

Pode ser verificado que os resultados obtidos de EF foram semelhantes tanto com a utilização do fundo geoquímico natural ou com a composição média da crosta terrestre, com exceção do Zn, cuja concentração na crosta terrestre foi superior ao fundo natural, causando uma diminuição do EF.

A Figura 3 ilustra a variabilidade do fator de enriquecimento dos metais pesados $\mathrm{Cr}, \mathrm{Cu}, \mathrm{Ni}, \mathrm{Zn}, \mathrm{Pb}$ ao longo do rio Tietê, desde a nascente até a barragem da Barra Bonita, evidenciando as contribuições naturais $(\mathrm{EF}<2)$ e antrópicas (EF > 2) de acordo com Sposito (1989). Tal classificação é um tanto subjetiva, pois alguns autores sugerem que para EF entre 2 e 5, as contribuições são consideradas moderadas, sendo superior a 5 a contribuição antrópica propriamente dita (Sutherland e Tolosa, 2000).

De qualquer maneira, os resultados de EF apresentados para o rio Tietê se mostraram expressivos e preocupantes, principalmente com relação ao $\mathrm{Zn}$ no trecho T2 a T4, após a influência antrópica da "grande São Paulo", onde os valores de EF foram cerca de 10 vezes o fundo geoquímico natural. Conforme observado na extração sequencial seletiva, mais de $60 \%$ do $\mathrm{Zn}$ se mostrou concentrado na fração biodisponível e principalmente ligado ao óxido de Fe. Concentrações elevadas de Cr também produziram EF superiores a 4, nessa mesma parte da bacia de drenagem, chegando próximo a 8 na represa da Barra Bonita. Da mesma forma como o $\mathrm{Zn}$, o $\mathrm{Cr}$ se mostrou associado ao óxido de $\mathrm{Fe}$, sendo $40 \%$ biodisponível.

\section{Índice de geoacumulação}

As classes de poluição dos sedimentos de fundo ao longo do rio Tietê foram determinadas em função do cálculo do índice de geoacumulação $\left(I_{\mathrm{geo}}\right)$ em cada estação de amostragem, considerando o fundo geoquímico natural para a série de metais estudados. A Tabela 4 apresenta o relacionamento entre $I_{\text {geo }}$ e as classes de poluição para os metais pesados $\mathrm{Cr}, \mathrm{Cu}, \mathrm{Ni}, \mathrm{Zn}, \mathrm{Pb}$ extraídos dos sedimentos de fundo ao longo do rio Tietê.

Com relação à parte mais a montante da bacia de drenagem, próximo às nascentes do rio Tietê ( $\mathrm{T} 0$ e $\mathrm{T} 1)$, 

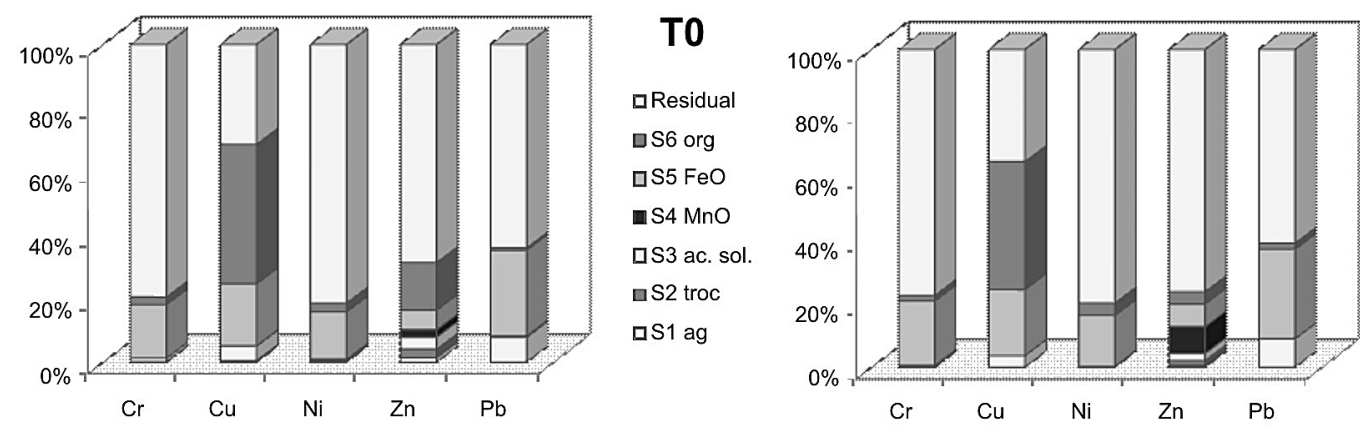

$\square$ Residual

$\square$ S6 org

$\square \mathrm{S} 5 \mathrm{FeO}$

- $\mathrm{S} 4 \mathrm{MnO}$

$\square$ S3 ac. sol.

$\square \mathrm{S} 2$ troc

$\square \mathrm{S} 1 \mathrm{ag}$
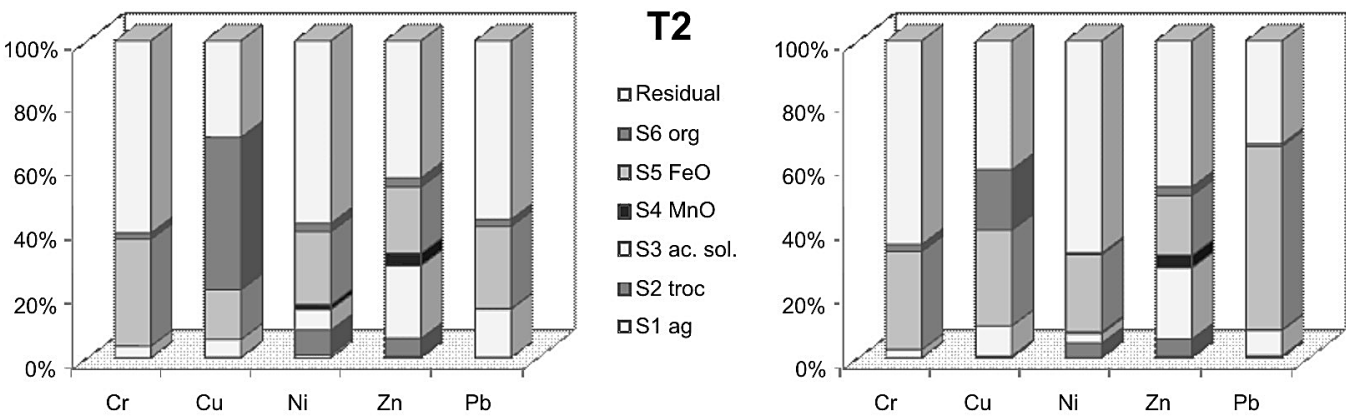

T3

$\square$ Residual $\square$ S6 org

$\square \mathrm{S} 5 \mathrm{FeO}$

ㄴ. $\mathrm{S} 4 \mathrm{MnO}$

口S3 ac. sol.

$\square \mathrm{S} 2$ troc

$\square \mathrm{S} 1 \mathrm{ag}$

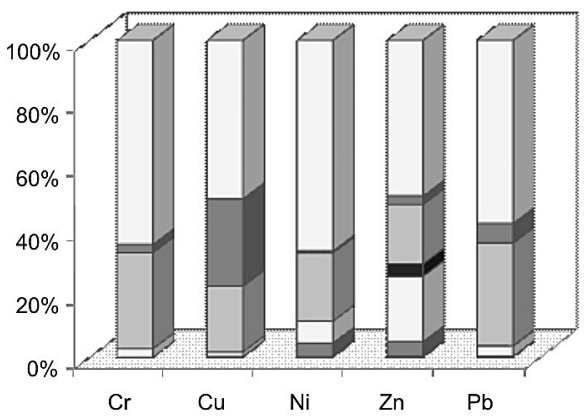

T4

口Residual

口S6 org

口S5 FeO

口S4 MnO

口S3 ac. sol.

$\square S 2$ troc

$\square \mathrm{S} 1 \mathrm{ag}$

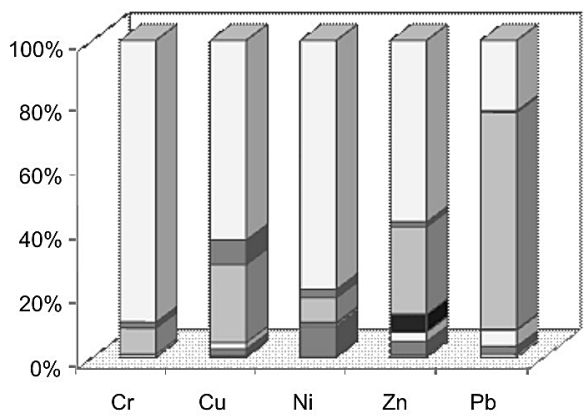

T5

口Residual

$\square$ S6 org

口S5 FeO

G $4 \mathrm{MnO}$

口S3 ac. sol.

$\square \mathrm{S} 2$ troc

口S1 ag
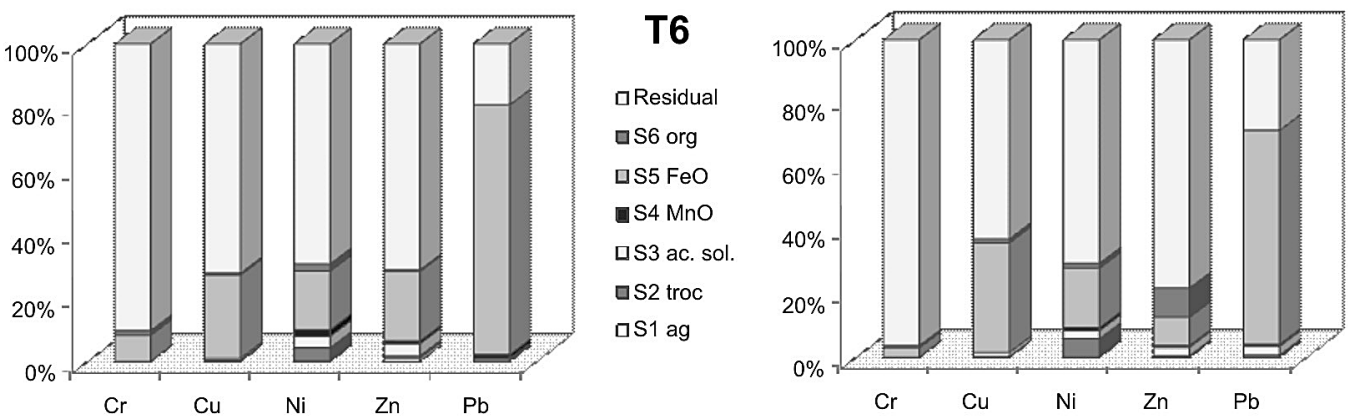

T7

$\square$ Residual

$\square$ S6 org

$\square \mathrm{S} 5 \mathrm{FeO}$

घ $4 \mathrm{MnO}$

口S3 ac. sol.

$\square \mathrm{S} 2$ troc

$\square \mathrm{S} 1 \mathrm{ag}$

Figura 2. Extração sequencial seletiva dos metais pesados presentes nos sedimentos de fundo ao longo do rio Tietê (T0. Junto à nascente em Salesópolis. T1. Mogi das Cruzes. T2. Santana do Parnaíba. T3. Tietê. T4. Anhembi. T5. Junto à entrada da represa de Barra Bonita. T6. No meio da represa de Barra Bonita. T7. Junto à barragem de Barra Bonita). 
Tabela 3. Fatores de enriquecimento (EF) para os metais estudados, calculados em função do fundo geoquímico natural da bacia de drenagem do rio Tietê e em relação à composição média da crosta terrestre.

\begin{tabular}{|c|c|c|c|c|c|c|c|c|c|c|}
\hline \multirow{2}{*}{ EF } & \multicolumn{2}{|c|}{$\mathrm{Cr}$} & \multicolumn{2}{|c|}{$\mathrm{Cu}$} & \multicolumn{2}{|c|}{$\mathbf{N i}$} & \multicolumn{2}{|c|}{$\mathrm{Zn}$} & \multicolumn{2}{|c|}{$\mathrm{Pb}$} \\
\hline & crosta & $\mathbf{F N}_{\text {sed }}$ & crosta & $\mathrm{FN}_{\text {sed }}$ & crosta & $\mathrm{FN}_{\text {sed }}$ & crosta & $\mathrm{FN}_{\text {sed }}$ & crosta & $\mathrm{FN}_{\text {sed }}$ \\
\hline TO & 1,2 & 1,3 & 1,1 & 0,7 & 1,5 & 1,3 & 0,9 & 1,5 & 1,6 & 1,3 \\
\hline $\mathrm{T} 1$ & 1,9 & 2,1 & 1,2 & 0,8 & 0,9 & 0,8 & 2,2 & 3,9 & 1,4 & 1,2 \\
\hline T2 & 3,0 & 3,3 & 4,5 & 2,9 & 2,6 & 2,4 & 5,2 & 9,4 & 2,2 & 1,8 \\
\hline T3 & 2,6 & 2,8 & 2,1 & 1,4 & 2,0 & 1,8 & 5,3 & 9,6 & 1,5 & 1,3 \\
\hline $\mathrm{T} 4$ & 2,8 & 3,1 & 2,9 & 1,9 & 2,5 & 2,3 & 4,1 & 7,4 & 1,4 & 1,2 \\
\hline T5 & 2,8 & 3,1 & 1,4 & 0,9 & 2,9 & 2,7 & 2,8 & 5,0 & 1,1 & 0,9 \\
\hline T6 & 2,4 & 2,7 & 1,4 & 0,9 & 3,1 & 2,9 & 2,1 & 3,7 & 0,8 & 0,7 \\
\hline 77 & 5,8 & 6,5 & 1,7 & 1,1 & 2,0 & 1,8 & 5,6 & 10,2 & 0,7 & 0,6 \\
\hline
\end{tabular}

$\square \mathrm{Cr} \square \mathrm{Cu} \square \mathrm{Ni} \square \mathrm{Zn}$ 田b

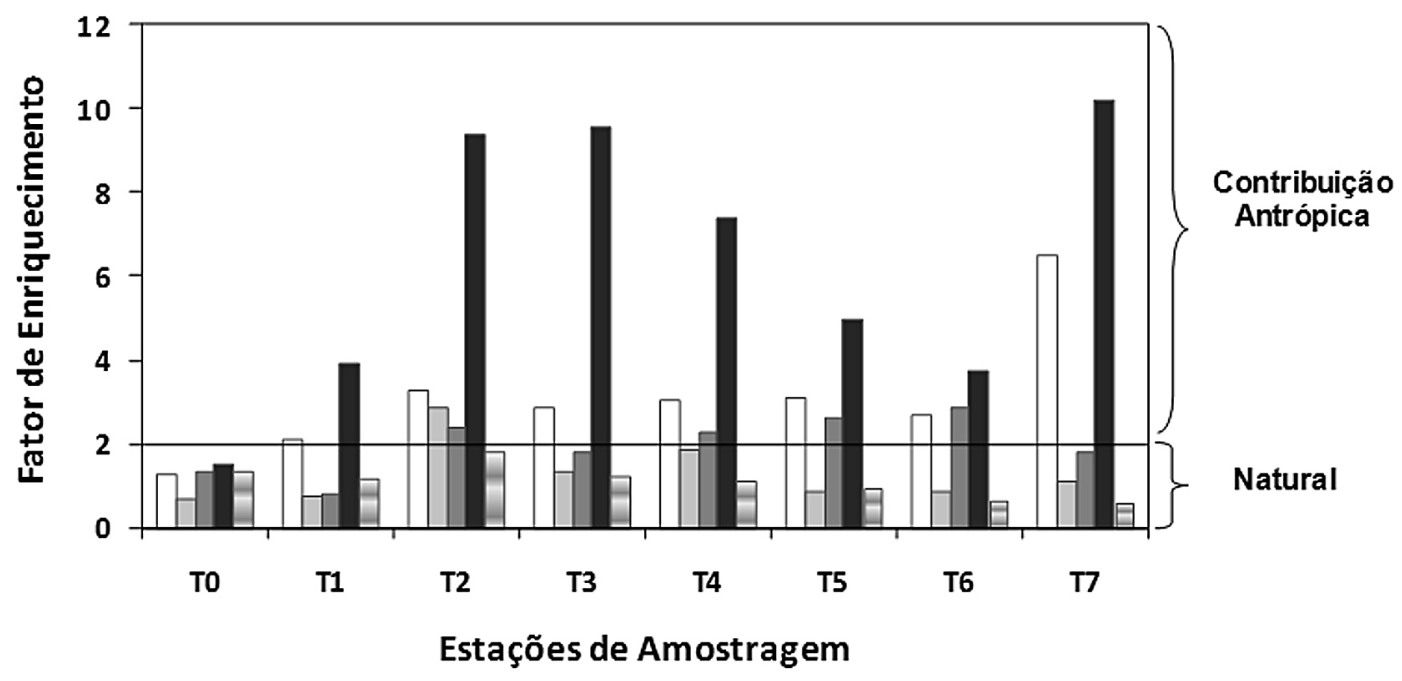

Figura 3. Variabilidade do fator de enriquecimento (EF) dos metais pesados estudados ao longo do rio Tietê, desde a nascente até a barragem de Barra Bonita.

as classes de poluição observadas foram ausentes e ausentes/moderadas para a maioria dos metais pesados, com exceção do Zn, que mesmo nessa região, se mostrou como poluição moderada. No trecho médio da bacia, após a influência urbana da capital paulista (T2 e $\mathrm{T} 3$ ), moderadas contribuições de $\mathrm{Cr}, \mathrm{Cu}$ e $\mathrm{Ni}$ puderam ser verificadas, enquanto que o $\mathrm{Pb}$ permaneceu praticamente ausente. Nesse mesmo ponto, o $\mathrm{Zn}$ atingiu a classe de poluição considerada forte. Tal ocorrência foi confirmada anteriormente pelo fator de enriquecimen- to e pelo caráter de biodisponibilidade após as extrações sequenciais.

Na parte baixa da bacia de drenagem, junto à represa de Barra Bonita (T7), Cr e Zn se mostraram dentro da classe de poluição moderada/forte e já com indícios de poluição ausente/moderada de Ni.

Os resultados obtidos revelam poluição moderada para a maioria dos metais pesados ao longo da bacia do rio Tietê, com maior preocupação para $\mathrm{Zn}$ e $\mathrm{Cr}$ a partir das contaminações derivadas de efluentes domésticos, agrícolas e industriais. 
Tabela 4. Índice de geoacumulação, $I_{\text {geo }}$ e as classes de polvição obtidas para os metais pesados extraídos dos sedimentos de fundo ao longo do rio Tietê.

\begin{tabular}{|c|c|c|c|c|c|c|c|}
\hline \multirow{3}{*}{$\begin{array}{c}\text { Local } \\
\text { T0 }\end{array}$} & \multirow{3}{*}{$\begin{array}{l}\mathrm{Cr} \\
<0\end{array}$} & \multirow{3}{*}{$\begin{array}{l}\mathrm{Cu} \\
<0\end{array}$} & \multirow{3}{*}{$\frac{\mathbf{N i}}{\frac{I_{\text {geo }}}{0-1}}$} & \multirow{3}{*}{$\begin{array}{l}\text { Zn } \\
0-1\end{array}$} & \multirow{3}{*}{$\begin{array}{c}\mathbf{P b} \\
0-1\end{array}$} & \multirow{2}{*}{\multicolumn{2}{|c|}{ Classes de Poluição }} \\
\hline & & & & & & & \\
\hline & & & & & & $<0$ & ausente \\
\hline $\mathrm{T} 1$ & $0-1$ & $<0$ & $<0$ & $1-2$ & $0-1$ & $0-1$ & ausente/moderada \\
\hline $\mathrm{T} 2$ & $1-2$ & $1-2$ & $1-2$ & $3-4$ & $0-1$ & $1-2$ & moderada \\
\hline T3 & $1-2$ & $0-1$ & $0-1$ & $2-3$ & $<0$ & $2-3$ & moderada/forte \\
\hline $\mathrm{T} 4$ & $1-2$ & $0-1$ & $0-1$ & $2-3$ & $<0$ & $3-4$ & forte \\
\hline T5 & $1-2$ & $<0$ & $0-1$ & $1-2$ & $<0$ & $4-5$ & forte/muito forte \\
\hline T6 & $0-1$ & $<0$ & $0-1$ & $1-2$ & $<0$ & $>5$ & muito forte \\
\hline $\mathrm{T} 7$ & $2-3$ & $<0$ & $0-1$ & $2-3$ & $<0$ & & \\
\hline
\end{tabular}

\section{CONCLUSÕES}

1. Elevadas concentrações de metais pesados nos sedimentos de fundo ao longo do rio Tietê puderam ser verificadas, principalmente $\mathrm{Zn}$ e $\mathrm{Cr}$, após a influência dos efluentes urbanos da cidade de São Paulo.

2. Os cálculos dos fatores de enriquecimento $\mathrm{EF}$ e os índices de geoacumulação identificaram o grau e as classes de poluição presentes na bacia de drenagem, sendo verificada para o caso do $\mathrm{Cr}$, uma poluição moderada/forte e para o $\mathrm{Zn}$, uma poluição já considerada forte.

3. Os resultados da extração sequencial seletiva confirmaram esse cenário preocupante de poluição dos sedimentos de fundo do rio Tietê, onde cerca de $60 \%$ do $\mathrm{Zn}$ extraído se mostrou na fração biodisponível, principalmente associado aos óxidos de $\mathrm{Fe}$, similar ao $\mathrm{Cr}$, com $40 \%$ também disponível.

\section{AGRADECIMENTOS}

Os autores agradecem a Fundação de Amparo à Pesquisa do Estado de São Paulo, FAPESP, pelo apoio financeiro, ao Conselho Nacional de Desenvolvimento Científico e Tecnológico, CNPq, pela bolsa de Produtividade em Pesquisa e a Universidade de São Paulo dentro do projeto USP/ COFECUB n ${ }^{\circ}$ 90/03 entre o Laboratoire des Mécanismes de Transfert en Géologie (LMTG - UMR 5563 CNRS/ Université Paul Sabatier) de Toulouse, França e o Centro de Energia Nuclear na Agricultura, CENA/USP.

\section{REFERÊNCIAS}

ELDERFIELD, H.; UPSTILL-GODDARD, R.; SHOLKOVITZ, E. R. The rare earth elements in rivers, estuaries and coastal seas and their significance to the composition of ocean water. Geochimica et Cosmochimica Acta, v. 54, p. 971-991, 1990.

FAVARO, P. C.; FERNANDES, E. A. N.; FERRAZ, E. S. B.; FALOTICO, M. H. B. Time still to restore the polluted Piracicaba river basin. Journal of Radioanalytical and Nuclear Chemistry, v. 2, p. 217-221, 2004.

FÖRSTNER, U.; SALOMONS, W. Trace metal analysis of polluted sediments, Part 1, assessment of sources and intensities. Environmental Technolog, v. 1, n. 11, p. 494-505, 1980.

GOMEZ, A. A.; VALENZUELA, J. L. G.; AGUAYO, S. S.; MEZA, D. F.; RAMIREZ, J. H.; OCHOA, G. O. Chemical partitioning of sediment contamination by heavy metals in the San Pedro River, Sonora, Mexico. Chemical Speciation and Bioavailability, v. 19, n. 1, p. 25-35, 2007.

GRESENS, R. L. Composition-volume relationships of metasomatism. Chemical Geology, v. 2, p. 47-55, 1967.

LELEYTER, L.; PROBST, J. L. A new sequential extraction procedure for the speciation of particulate trace elements in river sediments. International Journal of Environmental Analytical Chemistry, v. 73, n. 2, p. 109-128, 1999.

MOLISANI, M. M.; SALOMÃO, M. S. M. B.; OVALLE, A. R. C. Partitioning of metals in sediments from the lower Paraíba do sul river, SE, Brazil. Geochimica Brasiliensis, v. 19, n. 1, p. $48-59,2005$

MORTATTI, J.; BERNARDES, M. C.; PROBST, J. L.; LELEYTER-REINERT, L. Composição química dos se- 
dimentos fluviais em suspensão na bacia do rio Piracicaba: extração seletiva de elementos traço. Geochimica . Brasiliensis, v. 16, n. 2, p. 123-141, 2002.

MÜLLER, G. Schwermetalle in den sedimenten des rheins-veränderungen seit. Umschav. 79: 133-149, 1979.

OLIVEIRA J. B. Solos do Estado de São Paulo: descrição das classes registradas no mapa pedológico. Instituto Agronômico de Campinas, IAC, Boletim Científico, 45, 1999. $112 \mathrm{p}$.

SALOMONS, W.; FÖRSTNER, U. Metals in the hydrocycle. Berlin: Springer-Verlag, 1984. 349 p.

SAMUEL, J.; ROULT, R.; BESNUS, Y. Analyse multiélémentaire standardisée des matérieux géologiques en spectrométrie d'émission par plasma a couplage inductif. Analusis, v. 13, p. 312-317, 1985.

SHOLKOVITZ, E. R. Chemical evolution of rare earth elements: fractionation between colloidal and solution phases of filtered river water. Earth and Planetary Science Letters, v. 114, p. 77-84, 1992.

SPOSITO, G. The chemistry of soils. New York: Oxford University Press, 1989. 277 p.

SUTHERLAND, R. A.; TOLOSA, C. A. Multi-element analysis of road deposited sediment in an urban drainage basin, Honolulu, Hawaii. Environmental Pollution, v. 110, p. $483-495,2000$.

TAYLOR, S. R.; McLENNAN, S. M. The continental crust: Its composition and evolution. Blackwell: Oxford University Press, 1985. 312 p.

YAN, J. P.; YONG, H.; HUANG, H. Characteristics of heavy metals and their evaluation in sediments from middle and lower reaches of the Huaihe River. Journal of China University of Mining and Technology, v. 17, n. 3, p. 414-417, 2007. 